\title{
Timing Jitter Tolerant All-Optical TDM Demultiplexing using a Saw-Tooth Pulse Shaper
}

\author{
F. Parmigiani, T. T. Ng, M. Ibsen, P. Petropoulos, D. J. Richardson
}

\begin{abstract}
We experimentally demonstrate the generation of picosecond saw-tooth optical pulses, which are shaped using a fiber Bragg grating, to provide timing jitter tolerant optical time-division multiplexing (OTDM) data demultiplexing in a scheme based on cross-phase modulation (XPM) in an optical fiber, with subsequent offset filtering. A power improvement of more than $5 \mathrm{~dB}$ is obtained compared to demultiplexing with more conventional shapes, such as Gaussian, with a similar pulse width.
\end{abstract}

Index Terms - Optical pulse shaping, fiber Bragg grating, highly nonlinear fiber, cross-phase modulation, optical time division multiplexing, demultiplexing, triangular shape.

\section{INTRODUCTION}

A ll-optical time-division multiplexing technology has developed significantly over recent years and rates well in excess of 1Tbit/s have now been reported. Whilst multiplexing of tributary signals via passive interleaving is reasonably straightforward, demultiplexing of the data at appropriate points within a network represents a significant technical challenge due to the ultrafast optical switching required. Among the various demultiplexing schemes reported so far, (see e.g. [1-5]), the approach based on cross-phase modulation induced wavelength shifting in highly nonlinear fibers (HNLFs) is particularly attractive due to its relative simplicity [3].

According to this scheme short intense control pulses (at the tributary rate) are used to impart a frequency shift through XPM onto the desired tributary channel of a copropagating OTDM data signal so that it is pushed out of its initial spectral band. In particular, the OTDM data pulse overlapping with the leading (trailing) edge of the control pulse experiences a red (blue) shift of its spectrum. Demultiplexing is then completed by spectrally filtering the wavelength-shifted component to drop this OTDM tributary. The extent of the frequency-shift is proportional to the intensity-gradient experienced by each data bit due to its interaction with the control pulse. This is a function both of the relative timing of the control and data pulses, and of the control pulse shape itself. If a symmetric control pulse

This work was supported in part by the STREP TRIUMPH (IST027638). M. Ibsen acknowledges the support from a Royal Society University Research Fellowship.

The authors are with the Optoelectronics Research Centre, University of Southampton, SO17 1BJ, United Kingdom (e-mail: frp@orc.soton.ac.uk). is used, its width needs to be less than half of the OTDM bit slot and at least twice the signal pulse width to make the scheme work properly [3]. This implies that the signal pulse width has to be less than one quarter or so of the time slot available, which limits its scalability to higher bit rates. On the contrary, if an asymmetric control pulse is used, the system can be operated on its longer trailing/leading edge, so that far broader switching windows can be defined. This also ensures that the system is more resilient to temporal bit misalignment between the control and data signals. At the same time, most work in this area to date has used either Gaussian or soliton control pulses which exhibit significant variations in intensity gradient across their profile. Since both these shapes have a uniform gradient over only a very limited time, they provide a very narrow switching window resulting in acute timing sensitivity, reduced switching efficiencies and chirped output pulses. The optimum control pulses for such a demultiplexer have a saw-tooth profile [3], since the uniform gradient associated with this pulse shape gives rise to a constant frequency shift across its leading (trailing) edge. This ensures minimal distortion of the switched signal since the entire switched data pulse experiences the same frequency shift.

We have recently demonstrated the generation of $\sim 10 \mathrm{ps}$ saw-tooth (asymmetric triangular) pulses using superstructured fiber Bragg grating (SSFBG) technology [6], which allows the development of optical filters with accurately controlled frequency and phase responses of almost arbitrary complexity in a single, continuous grating structure [7]. Symmetric triangular pulses can also be generated in a normally dispersive HNLF by accurately pre-chirping the incoming pulse [8]. In this Letter we demonstrate a demultiplexing system that uses saw-tooth control pulses and highlight its timing jitter tolerance achieved relative to the use of the more conventional Gaussian pulses. Note that the preliminary results of this work were reported in [9].

\section{EXPERIMENTAL SET-UP AND RESULTS}

The experimental set-up is shown in Fig.1(a). The $~ 10 p s$ (full width at half maximum, FWHM) saw-tooth pulses are generated by feeding 1.3 ps Gaussian pulses at $10 \mathrm{GHz}$ 


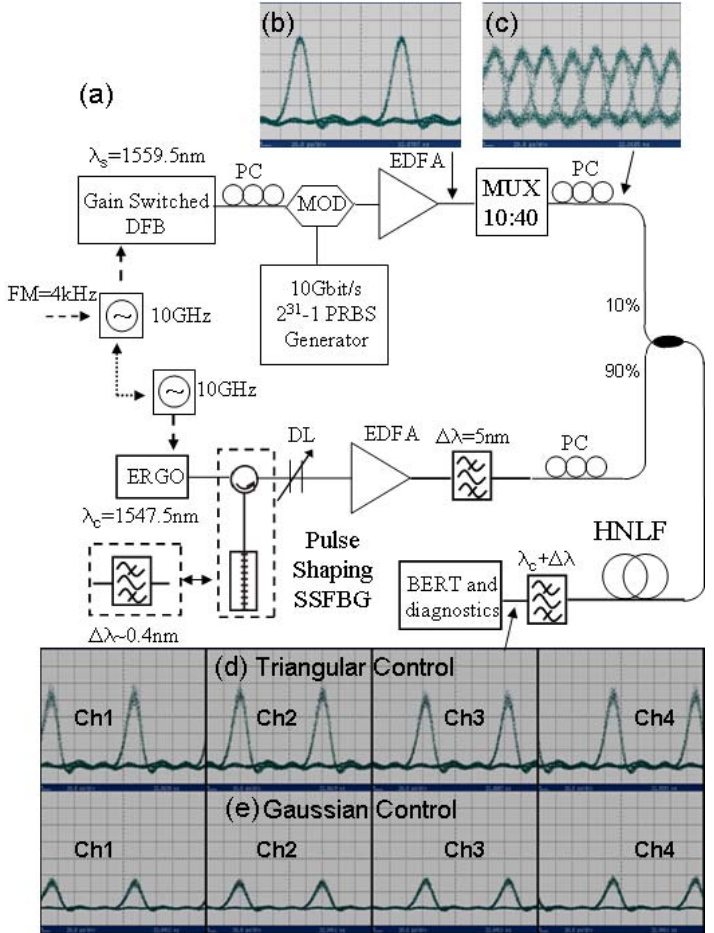

Figure 1. a) Experimental set-up of the demultiplexing system. ERGO: Erbium-glass oscillator laser. FM: frequency modulation, MOD: amplitude modulator, PRBS: pseudo-random bit sequence, EDFA: erbium doped fiber amplifier, BERT: Bit error rate tester, PC: polarisation controller, MUX: multiplexer. b-e) Eye diagrams at different points of the set-up.

to the specially designed SSFBG. The central wavelength of the SSFBG is $\sim 1547.5 \mathrm{~nm}$, its $-3 \mathrm{~dB}$ bandwidth is $\sim 0.4 \mathrm{~nm}$ and its peak reflectivity is $\sim 25 \%$, corresponding to a modulation index of $\sim 4 \times 10^{-4}$. The full characterization of the grating is reported in [6]. We characterized the shaped pulses using linear frequency-resolved optical gating (lFROG). The corresponding temporal profile is plotted in Fig. 2(a) together with a measurement of Gaussian pulses of similar FWHM. This shape was obtained simply by replacing the SSFBG with a suitable narrowband filter $(\Delta \lambda \sim 0.4 \mathrm{~nm})$. The saw-tooth pulses are then amplified to $\sim 25 \mathrm{dBm}$ and launched into a HNLF through a 90:10 coupler. The fiber characteristics are as follows: length of $310 \mathrm{~m}$, dispersion of $-0.31 \mathrm{ps} / \mathrm{nm} / \mathrm{km}$, dispersion slope of $0.0031 \mathrm{ps} / \mathrm{nm}^{2} / \mathrm{km}$, nonlinear coefficient of $22 / \mathrm{W} / \mathrm{km}$ and loss of $1.21 \mathrm{~dB} / \mathrm{km}$. A $40 \mathrm{Gbit} / \mathrm{s}$ data signal (Fig.1(c)) is then coupled into the HNLF using the $10 \%$ coupler port. This data signal is generated by passive interleaving a 10Gbit/s data signal (Fig.1(b)) generated from an externally modulated Gain switched distributed feedback (GS-DFB) laser providing $10 \mathrm{GHz}, 7 \mathrm{ps}$ pulses at $1559.5 \mathrm{~nm}$. The input power of the OTDM data was intentionally kept low ( $\sim \mathrm{dBm}$ at the input of the HNLF) to avoid any undesired self-phase modulation in the HNLF.

By appropriately adjusting the optical delay line (DL) in the control arm, it is possible to scan the saw-tooth pulse through any one of the four 10Gbit/s tributaries, thereby shifting the wavelength of the latter, and allowing demuxing
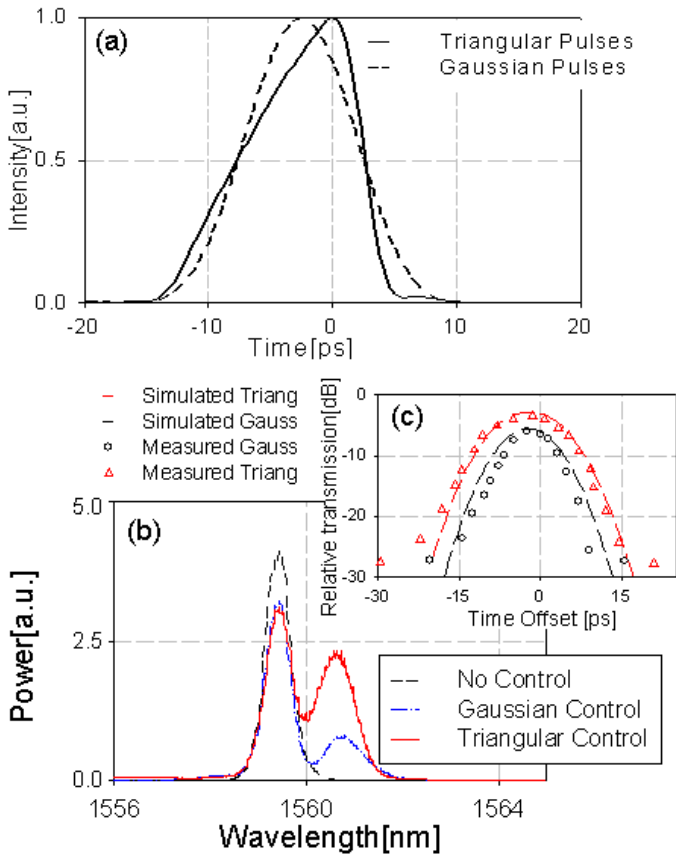

Figure 2. a) Saw-tooth and Gaussian intensity profiles. b) XPM broadened spectral traces of the data signal at the output of the HNLF. c) Simulated and measured drop windows for Gaussian and saw-tooth shapes.

to be implemented by simply placing a narrow band $(\sim 0.5 \mathrm{~nm})$ filter at the offset wavelength. Examples of the obtained spectra of the XPM wavelength-shifted pulses are shown in Fig.2(b) when either the saw-tooth or Gaussian pulses are used to control the switch. The OTDM data spectrum, when the control signal is turned off, is also shown as a reference. As expected, the spectral density of the wavelength-shifted component is much higher when using the saw-tooth control pulses; moreover the bandwidth is narrower due to the constant time-derivative of the pulse shape [6]. This implies that most of the energy of the wavelength-shifted drop channel passes through the offset filter (offset by $\sim 1.3 \mathrm{~nm}$ from the central wavelength of the data) and is efficiently detected. This effect is clearly highlighted in the drop windows for the two shapes (Fig.2(c)). These were obtained by varying the time delay between the control and data signals and measuring the corresponding output powers after the filter, when a $10 \mathrm{Gbit} / \mathrm{s}$ data signal was used at the input. Note that the drop windows are shown to have smooth edges, because of the comparable widths of the data and control pulses. Eye diagrams of a demultiplexed channel, when either sawtooth or Gaussian control pulses are used, are shown in Fig.1(d) and Fig.1(e) respectively, where a common vertical scale is used for the shapes to emphasize the difference in output power level and hence the optical signal to noise ratio (OSNR) obtained. Note, however, that for the same wavelength-shift the Gaussian pulses require $\sim 2 \mathrm{~dB}$ less average power compared to the saw-tooth pulses due to the fact that Gaussian pulses have a larger gradient, albeit more temporally localised, at their optimal operating point. To confirm the benefit of using saw-tooth control 
pulses in this demultiplexing scheme, we performed biterror rate (BER)

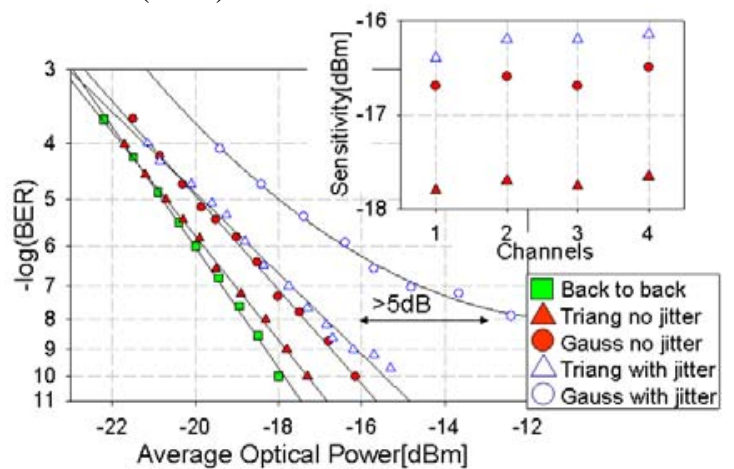

Figure 3. BER curves before and after the re-timing system when Gaussian (circles) or saw-tooth (triangular) control pulses are considered (Agilent 8343A receiver). Filled (empty) symbols correspond to no timing jitter ( 2.2ps rms timing jitter) added to the data signal. Inset: Corresponding receiver sensitivity for error free operation $\left(B E R=10^{-9}\right)$ when the two shapes are considered.

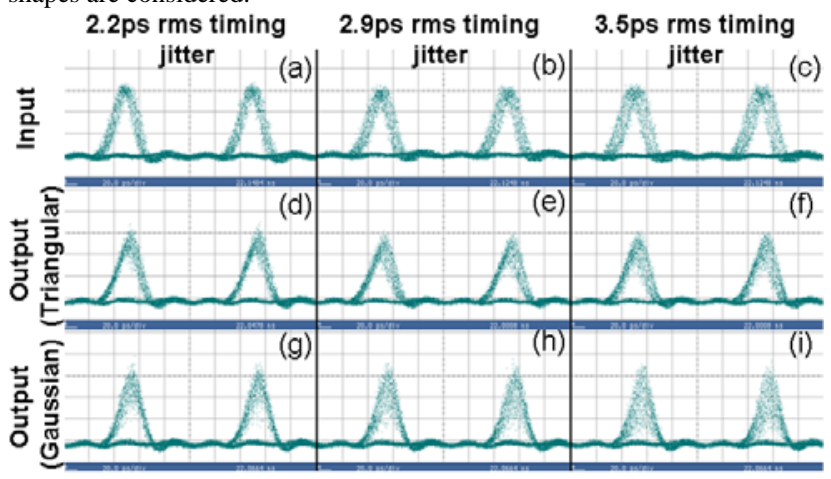

Figure 4. Eye-diagrams at different points of the set-up when different amount of timing jitter is induced onto the OTDM data.

measurements. At first, we measured BER curves for the demultiplexed channels for a clean OTDM signal, see Fig.3. Error free demultiplexing was achieved using sawtooth pulses (filled triangular symbols) for all the channels with a $\sim 0.5 \mathrm{~dB}$ penalty compared to the $10 \mathrm{Gbit} / \mathrm{s}$ back-toback (filled rectangular symbols). Error free operation was also achieved when using Gaussian pulses (filled circle symbols) albeit with a higher power penalty $(\sim 1.5 \mathrm{~dB})$. We believe that this extra penalty mainly rises from the inherent phase noise associated with the synthesizers used to drive the two laser sources. Indeed, much tighter tolerances on the timing between the control and data signals was required when Gaussian, rather than saw-tooth pulses, were used to control the demultiplexer. To further illustrate this point, we artificially introduced some timing jitter to the OTDM signal by frequency modulating the $10 \mathrm{GHz} \mathrm{RF}$ drive signal to the GS-DFB laser with a $4 \mathrm{kHz}$ tone (see Fig.1(a)). First, a 2.2ps root-mean-square (RMS) timing jitter was introduced (corresponding to 12.9ps peak-to-peak timing jitter ( $\tau p p)$ ), see eye diagram in Fig.4(a). When sawtooth pulses were used for demultiplexing, error-free operation was achieved for all OTDM channels (empty triangular symbols) with a penalty of less than $2 \mathrm{~dB}$ compared to the previous case (clean data). In contrast, an error floor was observed at a BER of $10-8$ and a power penalty of more than $5 \mathrm{~dB}$ was obtained for Gaussian pulses (empty circles). The eye-diagrams for the two cases are shown in Fig.4(d) and Fig.4(g) respectively, demonstrating that unlike the case of Gaussian pulses, the timing jitter is not converted into amplitude noise when saw-tooth shaped pulses are used. Finally, to study the limitations of our system, we increased further the timing jitter on the OTDM signal. The corresponding demultiplexed eye diagrams, when 2.9ps ( $\tau \mathrm{pp}=15.11 \mathrm{ps})$ and 3.5ps ( $\tau \mathrm{pp}=17.33 \mathrm{ps}) \mathrm{RMS}$ timing jitter was induced (Fig.4(b) and Fig.4(c), respectively), are reported in Fig.4(e)-(f) and Fig.4(h)-(i) for the two control shapes.

\section{CONCLUSION}

In conclusion, we have experimentally demonstrated the performance benefits of using saw-tooth shaped control pulses in a TDM demultiplexing system based on XPM in a HNLF and subsequent offset filtering. Error-free operation and significant improvement in the receiver sensitivity were achieved relative to the use of Gaussian control pulses of a similar width. Furthermore, we have shown that this scheme is more resilient to possible temporal bit misalignment between the control and the data signal and is hence more tolerant to timing jitter on the data signal.

We believe that this approach could be applied to much higher bit rate systems by properly scaling the width of the shaped pulses and the bandwidth and offset of the filter.

\section{ACKNOWLEDGEMENTS}

The authors acknowledge The Furukawa Electric Company, Japan for the loan of the HNLF.

\section{REFERENCES}

[1] A. I. Siahlo, A. T. Clausen, L. K. Oxenlowe, J. Seoane, P. Jeppesen, "640 Gb/s OTDM Transmission and Demultiplexing using a NOLM with Commercially Available Highly Non-linear Fiber" CLEO, (2005) paper CTuO1.

[2] V. Marembert, C. Schbert, C. Schmidt-Langhorst, M. Kroh, S. Ferber and H. G. Weber, "Investigation of fiber based gates for time division demultiplexing up to $640 \mathrm{Gbit} / \mathrm{s}$ ”, OFC, (2006) paper OWI12.

[3] J. Li, B.-E. Olsson, M. Karlsson, P. A. Andrekson, "OTDM AddDrop Multiplexer Based on XPM-Induced Wavelength Shifting in Highly Nonlinear Fiber”, IEEE J. Lightwave Technol., 23 (9), pp.2654-2661, 2005.

[4] M. D. Pelusi, “160-Gb/s Optical Time_Division Multiplexing Using a Mach-Zehnder Modulator in a fiber Loop”, IEEE Photon. Technol. Lett., 20 (12), pp.1060-1062, June 2008.

[5] D. M. Patrick, A. D. Ellis, "Demultiplexing using cross phase modulation induced spectral shifts and Kerr rotation in optical fibre", Elect Lett, Vol.29, No.2, pp 227, (1993).

[6] F. Parmigiani, M. Ibsen, T. T. Ng, L. Provost, P. Petropoulos, D. J. Richardson, "An Efficient Wavelength Converter Exploiting a Grating-Based Saw-Tooth Pulse Shaper”, IEEE Photon. Technol. Lett., 20 (17), pp.1461-1463, September 2008.

[7] P. Petropoulos, M. Ibsen, A. D. Ellis, and D. J. Richardson, "Rectangular pulse generation based on pulse reshaping using superstructured fiber Bragg grating,” J. Lightwave. Technol., vol. 19, no. 5, pp. 746-752, May 2001.

[8] A. I. Latkin, S. Bosco, S. K. Turitsyn, "Passive Nonlinear Pulse Shaping in Normally Dispersive Fiber”, OFC, (2008) paper OTu B7.

[9] F. Parmigiani, T. T. Ng, M. Ibsen, P. Petropoulos, D. J. Richardson, "Timing jitter tolerant OTDM demultiplexing using a saw-tooth pulse shaper,” ECOC (2008), paper Tu.4.B.5. 